\title{
Ocular Autonomic Nerve Function in Proliferative Diabetic Retinopathy
}

\author{
CHARLES V. CLARK \\ Edinburgh
}

\begin{abstract}
Summary
Ocular autonomic nerve function was assessed in 28 diabetic patients with proliferative retinopathy (PDR) and 61 age- and sex-matched control subjects, by measurement of the pupil cycle time and determination of autonomic denervation hypersensitivity of the iris. Sustained pupil cycle time was absent in $\mathbf{8 8 . 5}$ per cent of PDR compared with 9.8 per cent of control subjects $(p<0.001)$. Pupil constriction in response to 2.5 per cent methacholine—indicative of parasympathetic denervation hypersensitivity - was significantly increased in PDR $(p<0.001)$, whilst pupil dilation in response to 0.5 per cent phenylephrine-indicative of sympathetic denervation hypersensitivity - was also significantly higher in PDR $(p<0.001)$. Pupil reflexes were abnormal in 88.5 per cent of diabetics with proliferative retinopathy, with established autonomic denervation hypersensitivity in 57 per cent of patients.
\end{abstract}

The principle of denervation hypersensitivity-defined as an increased response of a tissue to a chemical neurotransmitter or agonişt following deprivation of its nerve supply 1 - may be applied in the assessment of ocular autonomic nerve function. Pupil diameter represents the relative balance of autonomic activity in the anterior segment of the eye; sympathetic nerves effect pupillary dilation via the dilator pupillae and parasympathetic nerves effect pupillary constriction via the sphincter pupillae. The assessment of parasympathetic denervation hypersensitivity is facilitated by measurement of the pupil diameter after topical application of 2.5 per cent methacholine, a parasympathetic agonist, which constricts Adie's tonic pupils whilst normal pupils may remain unaffected by concentrations of 15 per cent. ${ }^{2,3}$ Concentrations of sympathetic agonists diagnostic of sympathetic denervation hypersensitivity have not been definitively established; 1 to 2 per cent phenylephrine $^{4,5}$ and 0.1 per cent adrenaline ${ }^{6}$ have been proposed for this purpose, although the latter drug has given conflicting results. ${ }^{4}$ Assessment of ocular autonomic function is augmented by measurement of pupil cycle time, which enables the integrity of the pupillary reflex arc to be quantified accurately and objectively by a simple, noninvasive technique. ${ }^{7}$ In subjects with normal iris musculature, pupil cycle time is directly dependent upon nerve conduction velocity, synaptic delay, and the number of nerve impulses transmitted.

The aim of this study was to assess and quantify ocular autonomic nerve function in diabetic patients with proliferative retino-

From: Department of Ophthalmology, University of Edinburgh, Princess Alexandra Eye Pavilion, Edinburgh and St Paul's Eye Hospital, Old Hall Street, Liverpool.

Correspondence to: Mr C. V. Clark, MD, FRCS, Moorfields Eye Hospital, City Road, London EC1V 2PD. 
pathy by a series of tests based upon pupil responses to light, and to autonomic mediators recognised to be effective in the determination of ocular autonomic denervation hypersensitivity.

\section{Patients and Methods}

Ocular autonomic nerve function was assessed in 28 diabetic patients with proliferative retinopathy (mean aged $50.8 \pm 14.3$ years): 21 IDDM (mean age $46.0 \pm 12.9$ years) and 7 NIDDM (mean age $65.8 \pm 6.5$ years). Mean duration of diabetes was $20.9 \pm$ 8.8 years: IDDM (23.6 \pm 7.3 years); NIDDM $(12.6 \pm 7.9$ years). They were compared with a control group of 61 age- and sex-matched subjects (mean age 53.1 \pm 10.1 years) who were drawn from fit hospital staff and patients attending an ophthalmic casualty department subsequently determined to have no detectable abnormality. No subject had any medical disorder or were taking any medication known to interfere with autonomic function. Any individual eye was excluded if there was a history of trauma, disease, or operation on the eye, or current treatment for any eye disorder. The study was approved by the local Hospital Advisory Ethical Committee, and each patient was informed of the details of the tests.

\section{(i) Pupil cycle time}

Edge-light pupil cycle time was measured according to the following method. The patient was seated at a slit-lamp (Zeiss 30SL, Carl Zeiss, Welwyn Garden City, England) in a sealed room with low, constant background illumination (20 lux), and requested to fixate straight ahead on a distant object at 6 metres. A $0.5 \times 6 \mathrm{~mm}$ slit-beam was positioned horizontally and elevated until it just overlapped the inferior margin of the pupil, then focused on the iris-lens border. The pupil constricted, then reflexly dilated in response to removal of afferent stimulation by iris blocking the light. Thirty consecutive pupil cycles were timed by stop-watch; the result divided by 30 is the duration of a single cycle of the pupillary reflex arc. The pupil cycle time was measured 4 times for each eye separately (i.e. $4 \times$ 30 -cycles) and the result averaged. (ii) Autonomic denervation hypersensitivity

(a) Parasympathetic nerves

Pupil diameters were recorded photographically under standardised lighting conditions (luminance 20 apostilbs) using an ophthalmic head-rest and a scale positioned against the lower eyelid in the perpendicular plane of the iris. One drop of 2.5 per cent methacholine chloride solution was placed in the conjunctival sac of one eye, and one drop of sterile normal saline solution concurrently placed in the conjunctival sac of the other eye. The choice of the eye to be tested was selected randomly. Forty-five minutes later, a second photograph was taken.

\section{(b) Sympathetic nerves}

One month later, the assessment of sympathetic denervation hypersensitivity was performed using the same technique. A pupil photograph was taken, as described. One drop of 0.5 per cent phenylephrine hydrochloride was placed in the conjunctival sac of the previously untested eye and one drop of sterile normal saline solution placed in the conjunctival sac of the other eye. A second pupil photograph was taken forty-five minutes later.

The photographic slides were projected on to a white screen at five metres, producing a final magnification of $\times 17$. Horizontal pupil diameters were measured to an accuracy of $\pm 0.5 \mathrm{~mm}$; these were corrected to actual values by comparison with relative magnification of the scale. Measurements of actual pupil diameters were therefore accurate to $\pm 0.03 \mathrm{~mm}$.

Parasympathetic denervation hypersensitivity was expressed as the 2.5 per cent methacholine ratio:

horizontal pupil diameter 45 minutes post-test

horizontal pupil diameter pre-test

Sympathetic denervation hypersensitivity was measured in a similar manner, and expressed as the 0.5 per cent phenylephrine ratio:

horizontal pupil diameter 45 minutes post-test horizontal pupil diameter pre-test

Assessments were performed on a double- 
masked basis, with neither the examiner nor the subject being aware which was the tested eye.

Renal function was assessed by plasma creatinine ${ }^{8}$ and long-term glycaemic control was determined by measurement of glycosylated haemoglobin, using a micro-column method. ${ }^{9}$

\section{Statistical analysis}

Comparisons were made between the results of the control group and diabetic patients with proliferative retinopathy; significance was assessed by Student's unpaired t test, and $\chi^{2}$ test with Yates' correction. Results are expressed as mean \pm SEM.

\section{Results}

\section{(i) Pupil cycle time}

Sustained pupillary oscillation for 120 consecutive cycles in response to edge-light stimulation was absent in a significant percentage of eyes examined; pupil cycle time was absent in 88.5 per cent of diabetics with proliferative retinopathy compared with 9.8 per cent of control subjects $(\mathrm{p}<0.001)$. Consequently, measurement of pupil cycle time was possible in a proportionately decreased number of eyes: PDR 11.5 per cent; control group 90.2 per cent. There were no significant differences in pupil cycle time between those diabetics in whom pupil cycle time was present $(1,063 \pm 49 \mathrm{~ms})$ and the control group (971 $\pm 14 \mathrm{~ms})$, however this represents only 11.5 per cent of the diabetic patients.

\section{(ii) Parasympathetic denervation}

\section{hypersensitivity}

2.5 per cent methacholine ratios were significantly lower in diabetic patients with proliferative retinopathy $(0.84 \pm 0.03)$ than the control group $(0.95 \pm 0.01)(\mathrm{p}<0.001)$. Pupillary constriction resulting from methacholine stimulation of the sphincter pupillae in a diabetic patient with proliferative retinopathy is shown in Figure 1.

Age-adjusted normal tolerance intervals have been established for 2.5 per cent methacholine ratio; ${ }^{10} \log _{10}$ (2.5 per cent methacholine ratio) depends significantly on age ( $r$
$=-0.40 ; \mathrm{p}<0.001)$. The age-adjusted normal tolerance limit, arbitrarily set at the lower 5th percentile of the log normal distribution after regression on age, was applied to the results of diabetics with proliferative retinopathy, to determine the proportion of subjects outwith the normal limit. By this criterion, parasympathetic neuropathy was present in 36 per cent of diabetics with proliferative retinopathy, compared with 6.4 per cent of the control group.

(iii) Sympathetic denervation hypersensitivity 0.5 per cent phenylephrine ratios were significantly higher in diabetic patients with proliferative retinopathy $(1.35 \pm 0.05)$ than the control group $(1.17 \pm 0.03)(\mathrm{p}<0.001)$. Pupil dilation following phenylephrine stimulation of the dilator pupillae in a diabetic with proliferative retinopathy is shown in Figure 2.

Age-adjusted normal tolerance intervals for pupil response to 0.5 per cent phenylephrine have not been previously defined; 0.5 per cent phenylephrine ratios in the control group were therefore assessed for dependence on age by regression analysis. The data were fitted according to various mathematical models using the computer programme SPSSx to obtain a normal distribution on age, thereby permitting determination of tolerance intervals." Linear regression analysis was calculated from:

$\mathrm{RLP}_{0.5}=\left(\log _{10} \mathrm{P}_{0.5}-(-0.05084+0.00213 \times\right.$ age) ) $/ 0.05214$

RLP $_{0.5}=$ standardised residual log $(0.5$ per cent phenylephrine ratio)

$\log _{10}(0.5$ per cent phenylephrine ratio) was shown to depend significantly on age $(r=$ 0.64; p<0.001) (Fig. 3). The age-adjusted normal tolerance limit, arbitrarily set at the upper 95th percentile of the log normal distribution after regression on age, was then applied to the results obtained from diabetics with proliferative retinopathy, to determine the proportion of subjects outwith the normal limit. By this criterion, sympathetic neuropathy was present in 38.4 per cent of diabetics with proliferative retinopathy compared with 5.0 per cent of the control group.

Serum creatinine levels were not excessively abnormal in the diabetic group (104.5 \pm 


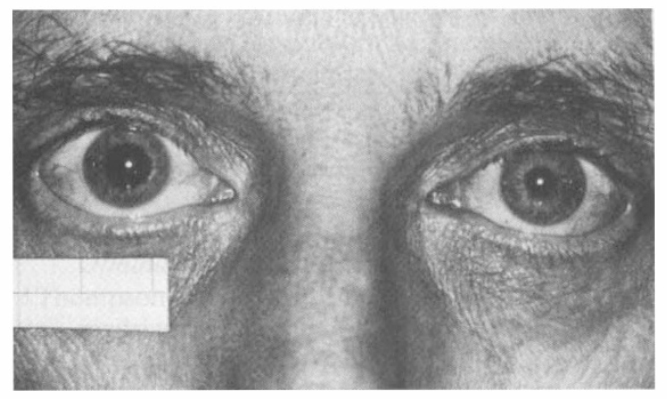

Fig. 1. Pupil constriction following instillation of 2.5 per cent methacholine chloride solution into the left eye of a diabetic patient with proliferative retinopathy, demonstrating parasympathetic denervation hypersensitivity.

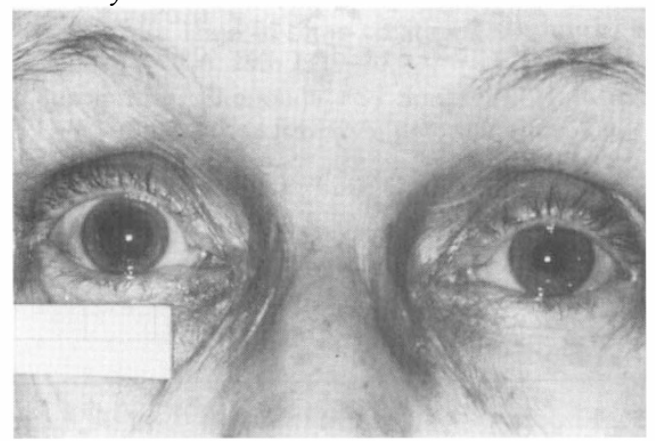

Fig. 2. Pupil dilation following instillation of 0.5 per cent phenylephrine hydrochloride solution into the right eye of a diabetic patient with proliferative retinopathy, demonstrating sympathetic denervation hypersensitivity.

$8.31 \mu \mathrm{mol} / \mathrm{l}$; normal range 55-110 $\mu \mathrm{mol} / \mathrm{l})$, with abnormal results in only 5 diabetic patients. Glycosylated haemoglobin was moderately elevated in diabetics with proliferative retinopathy $(10.3 \pm 0.42$ per cent; normal range $6.0-8.5$ per cent).

\section{Discussion}

Providing its structural integrity is intact, the iris facilitates direct measurement of autonomic efficacy in the anterior segment of the eye. Pupil cycle time permits rapid and accurate assessment of the pupillary reflex arc; the procedure is without side-effects and involves no expense, and is therefore directly applicable to the clinical situation. Recent studies have established that pupil cycle time is a particularly sensitive measure of dysfunction in the parasympathetic efferent limb of the pupillary reflex arc, ${ }^{12}$ however definitive anatomical localisation of defects requires more specific tests; this is provided by isolation of the primary determinants of pupil diameter-the sympathetic and parasympathetic nervous systems-by using minute concentrations of chemical agonists which only effect changes in pupil diameter in the presence of increased receptor density at the postsynaptic receptor site $^{13,14}$ - a characteristic feature of autonomic denervation hypersensitivity. Impaired parasympathetic innervation of the anterior segment is demonstrated by 2.5 per cent methacholine ${ }^{2.3}$; as responses to methacholine are significantly age-related, comparison with an age-adjusted normal tolerance interval is essential. The type and optimal concentration of agonist to be utilised in the assessment of sympathetic denervation hypersensitivity has not been established, however phenylephrine, a direct-acting sympathomimetic amine, which effects mydriasis by alpha-adrenoceptor stimulation of the sympatheticallyinnervated dilator pupillae,${ }^{15}$ is ideally suited for this specific purpose. The exact concentration of phenylephrine to be used in the evaluation of sympathetic denervation hypersensitivity is not as important as comparison with an age-adjusted normal tolerance interval; this has been established for 0.5 per cent phenylephrine, which may therefore be applied effectively in this context.

The results of the present study have shown significant ocular autonomic dysfunction in diabetic patients with proliferative retinopathy, thereby providing further evidence of an association between autonomic neuropathy and proliferative diabetic retinopathy. Parasympathetic neuropathy was present in 36 per cent of diabetics, with sympathetic neuropathy in 38.4 per cent of subjects, compared with a prevalence in the control group of 6.4 per cent and 5 per cent respectively. Ocular autonomic neuropathy involving either parasympathetic or sympathetic nerves was present in 57 per cent of diabetics compared with only 6.4 per cent of control subjects. Abnormalities in the pupillary reflex arc, with impairment or absence of sustained pupil cycling in response to light, was present in 88.5 per cent of diabetics with proliferative retinopathy; this may represent the earliest manifestation of ocular autonomic nerve dysfunction. One may reasonably postulate 


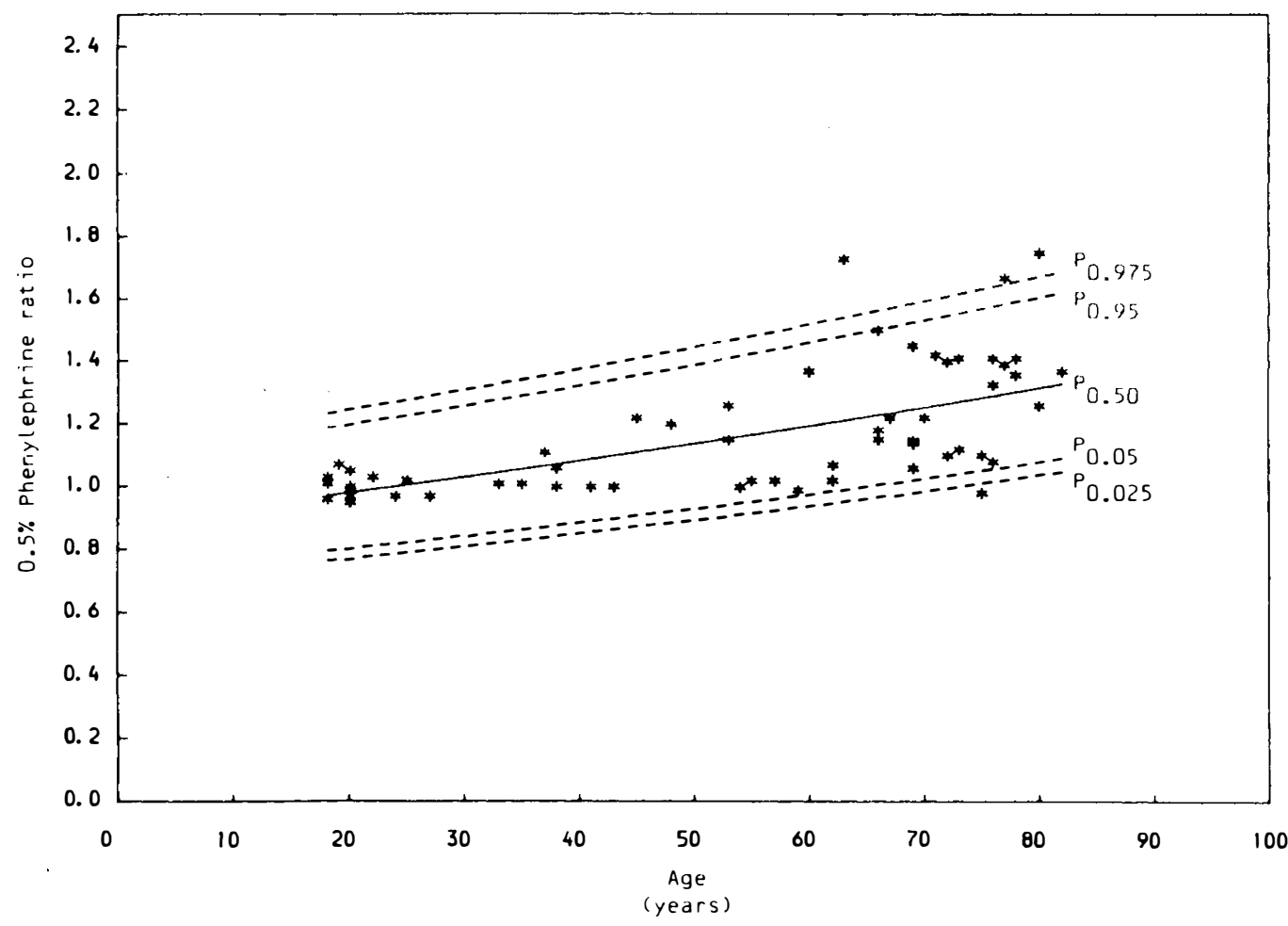

Fig. 3. Age-adjusted normal tolerance intervals for 0.5 per cent phenylephrine ratio, with 2.5 th, 5 th, 95 th, and 97.5th percentiles.

that assessment of iris dynamic function over prolonged periods of stimulation is more likely to reveal the prodromal signs of impaired efficacy prior to the development of specific changes in iris structure-for example, increased density of postsynaptic receptors, which is primarily determined by tests of denervation hypersensitivity.

Autonomic neuropathy is not characteristically a localised disorder. In diabetes, tests of ocular autonomic function correlate closely with autonomic function tests based upon cardiovascular reflexes. ${ }^{12}$ As systemic cardiovascular autonomic neuropathy has been demonstrated in 75 per cent of diabetics with proliferative retinopathy,$^{16}$ the presence of ocular autonomic neuropathy in 57 per cent of these patients is not surprising. Glycaemic control and renal function were not excessively abnormal in this group, duration of diabetes was not unduly prolonged, and therefore this is unlikely to represent a direct manifestation of severity of diabetes.

Previous studies have established a significant association between cardiovascular autonomic neuropathy and proliferative diabetic retinopathy, ${ }^{16.17}$ with direct correlation between the degree of cardiovascular autonomic neuropathy and the severity of retinopathy. ${ }^{17}$ Evidence of severe impairment of ocular autonomic nerve function in diabetics with proliferative retinopathy raises the inevitable question of whether this association, which cannot be explained exclusively in terms of diabetic severity, represents merely a coincidental natural history, ${ }^{17}$ or perhaps a more significant link between these diabetic complications.

I would like to acknowledge the help and support that 1 received from the late $\mathrm{Mr} \mathrm{R}$ Mapstone of $\mathrm{St}$ Paul's Eye Hospital, Liverpool. 


\section{References}

${ }^{1}$ Cannon WB and Rosenbluth A: The supersensitivity of denervated structures: a law of denervation. New York: Macmillan, 1949.

${ }^{2}$ Scheie HG: Site of disturbance in Adie's syndrome. Arch Ophthalmol 1940, 24: 225-37.

${ }^{3}$ Scheie HG and Adler FH: Site of disturbance of tonic pupils (Adie's syndrome). Arch Ophthalmol 1940, 24: 1041-4.

${ }^{4}$ Thompson HS and Mensher JH: Adrenergic mydriasis in Horner's syndrome. Am J Ophthalmol 1971, 72: 472-80.

${ }^{5}$ Smith SA: Horner's syndrome in diabetes mellitus. Diabetic Medicine (in press).

6 Jaffe NS: Localisation of lesions causing Horner's syndrome. Arch Ophthalmol 1950, 44: 710-28.

${ }^{7}$ Miller SD and Thompson HS: Edge-light pupil cycle time. Br J Ophthalmol 1978, 62: 495-500.

${ }^{8}$ Gabriel R: Time to scrap creatinine clearance? $\mathrm{Br}$ Med J 1986, 293: 1119-20.

${ }^{9}$ Baron MD, Shenouda FS, Sonksen PH: Microcolumn method for $\mathrm{HbA}_{1}$ determination. Lancet 1980 , i: $1114-16$.
10 Clark CV and Mapstone R: Parasympathetic denervation hypersensitivity of the iris in ocular hyper-. tension. Invest Ophthalmol Vis Sci (in press).

${ }^{11} \mathrm{Nie}$ NH: A complete guide to SPSSx language and operations. New York: McGraw Hill, 1983.

12 Martyn CN and Ewing DJ: Pupil cycle time: a simple way of measuring an autonomic reflex. $J$ Neurol Neurosurg Psychiatry 1986, 49: 771-4.

${ }_{13}$ U'Pritchard DC, Bechtel WD, Roust BM, Snyder SH: Multiple apparent alpha-noradrenergic binding sites in rat brain: effect of 6-hydroxydopamine. Molec Pharmacol 1979, 16: 47-60.

${ }^{14}$ U'Pritchard DC and Snyder SH: Increase in alphareceptor number in reserpine sensitivity in rats. Eur J Pharmacol 1978, 51: 145-55.

15 Turner P: The human eye as a target to analyse the mechanism of action of substances. Sandoz J Med Sci 1969, 3: 91-7.

${ }^{16}$ Clark CV: Autonomic neuropathy in proliferative diabetic retinopathy. Eye (in press).

${ }^{17}$ Smith SE, Smith SA, Brown PM: Cardiac autonomic dysfunction in patients with diabetic retinopathy. Diabetologia 1981, 21: 525-8. 